\title{
Measurement of the weak dipole moments of the $\tau$ lepton
}

\section{L3 Collaboration}

M. Acciarri ${ }^{\text {aa }}$, O. Adriani ${ }^{\text {p }}$, M. Aguilar-Benitez ${ }^{\mathrm{z}}$, S. Ahlen ${ }^{\mathrm{k}}$, J. Alcaraz ${ }^{\mathrm{z}}$,

G. Alemanni v , J. Allaby ${ }^{q}$, A. Aloisio ${ }^{\text {ac }}$, M.G. Alviggi ${ }^{\text {ac }}$, G. Ambrosi ${ }^{\text {s, }}$ H. Anderhub aw, V.P. Andreev al, T. Angelescu ${ }^{\mathrm{m}}$, F. Anselmo ', A. Arefiev ${ }^{\text {ab }}$, T. Azemoon ${ }^{\text {c }}$, T. Aziz ${ }^{j}$, P. Bagnaia ak ${ }^{\text {a }}$ L. Baksay ${ }^{\text {ar }}$, R.C. Ball ${ }^{c}$, S. Banerjee ${ }^{j}$, Sw. Banerjee ${ }^{j}, K$. Banicz ${ }^{\text {at }}$, A. Barczyk ${ }^{\text {aw,au }}$, R. Barillère ${ }^{q}$, L. Barone ${ }^{\text {ak }}$, P. Bartalini ah, A. Baschirotto ${ }^{\text {aa }}$, M. Basile ${ }^{\mathrm{i}}$, R. Battiston ${ }^{\text {ah }}$, A. Bay ${ }^{\mathrm{v}}$, F. Becattini ${ }^{\mathrm{p}}$, U. Becker ${ }^{\circ}$, F. Behner ${ }^{\text {aw }}$, J. Berdugo ${ }^{\text {z }}$, P. Berges ${ }^{\circ}$, B. Bertucci ${ }^{\text {ah }}$, B.L. Betev ${ }^{\text {aw }}$,

S. Bhattacharya ${ }^{\mathrm{j}}$, M. Biasini ${ }^{\mathrm{q}}$, A. Biland ${ }^{\text {aw }}$, G.M. Bilei ${ }^{\text {ah }}$, J.J. Blaising ${ }^{\mathrm{d}}$, S.C. Blyth ${ }^{\text {ai }}$, G.J. Bobbink ${ }^{\text {b }}$, R. Bock ${ }^{\text {a }}$, A. Böhm ${ }^{\text {a }}$, L. Boldizsar ${ }^{\text {n }}$, B. Borgia ${ }^{\text {ak }}$,

D. Bourilkov aw , M. Bourquin s, D. Boutigny ${ }^{\text {d }, ~ S . ~ B r a c c i n i ~}{ }^{\text {s, J.G. Branson an }}{ }^{\text {, }}$ V. Brigljevic aw , I.C. Brock ${ }^{\text {ai }}$, A. Buffini ${ }^{\mathrm{p}}$, A. Buijs ${ }^{\text {as }}$, J.D. Burger ${ }^{0}$, W.J. Burger ${ }^{\mathrm{s}}$,

J. Busenitz ${ }^{\text {ar }}$, X.D. Cai ${ }^{\circ}$, M. Campanelli ${ }^{\text {aw }}$, M. Capell ${ }^{\circ}$, G. Cara Romeo ${ }^{\text {, }}$,

G. Carlino ${ }^{\text {ac }}$, A.M. Cartacci p , J. Casaus ${ }^{\text {z }}$, G. Castellini ${ }^{\text {p }}$, F. Cavallari ${ }^{\text {ak}}$, N. Cavallo ${ }^{\text {ac }}$, C. Cecchi ${ }^{\text {s }}$, M. Cerrada ${ }^{\text {z }}$, F. Cesaroni ${ }^{\text {w }}$, M. Chamizo ${ }^{\text {z }}$, Y.H. Chang ay , U.K. Chaturvedi ${ }^{\mathrm{r}}$, S.V. Chekanov ${ }^{\text {ae }}$, M. Chemarin ${ }^{\mathrm{y}}$, A. Chen ${ }^{\text {ay }}$, G. Chen ${ }^{\mathrm{g}}$, G.M. Chen ${ }^{\mathrm{g}}$, H.F. Chen ${ }^{\mathrm{t}}$, H.S. Chen ${ }^{\mathrm{g}}$, M. Chen ${ }^{\circ}$, G. Chiefari ${ }^{\mathrm{ac}}$, C.Y. Chien ${ }^{\mathrm{e}}$, L. Cifarelli ${ }^{\mathrm{am}}$, F. Cindolo ${ }^{\mathrm{i}}$, C. Civinini ${ }^{\mathrm{p}}$, I. Clare ${ }^{\circ}$, R. Clare ${ }^{\circ}$, H.O. Cohn af , G. Coignet ${ }^{\text {d }}$, A.P. Colijn ${ }^{\text {b }}$, N. Colino ${ }^{\mathrm{z}}$, S. Costantini ${ }^{\mathrm{h}}$, F. Cotorobai ${ }^{\mathrm{m}}$, B. de la Cruz ${ }^{\mathrm{z}}$, A. Csilling ${ }^{\mathrm{n}}$, T.S. Dai ${ }^{\mathrm{o}}$, R. D'Alessandro ${ }^{\mathrm{p}}$, R. de Asmundis ${ }^{\text {ac }}$, A. Degré ${ }^{d}$, K. Deiters ${ }^{\text {au }}$, P. Denes ${ }^{\text {aj }}$, F. DeNotaristefani ${ }^{\text {ak }}$, D. DiBitonto ${ }^{\text {ar }}$, M. Diemoz ${ }^{\text {ak }}$, D. van Dierendonck ${ }^{\text {b }}$, F. Di Lodovico ${ }^{\text {aw }}$, C. Dionisi ${ }^{\text {ak }}$, M. Dittmar ${ }^{\text {aw }}$, A. Dominguez ${ }^{\text {an }}$, A. Doria ${ }^{\text {ac }}$, M.T. Dova ${ }^{\mathrm{r}, \mathrm{l}}$, E. Drago ${ }^{\text {ac }}$, D. Duchesneau ${ }^{\text {d }}$, P. Duinker ${ }^{b}$, I. Duran ${ }^{\text {ao }}$, S. Dutta ${ }^{j}$, S. Easo ${ }^{\text {ah }}$, Yu. Efremenko ${ }^{\text {af }}$, H. El Mamouni ${ }^{y}$, A. Engler ${ }^{\text {ai }}$, F.J. Eppling ${ }^{o}$, F.C. Erné ${ }^{b}$, J.P. Ernenwein ${ }^{y}$, P. Extermann ${ }^{\text {s }}$, M. Fabre ${ }^{\text {au }}$, R. Faccini ak, S. Falciano ${ }^{\text {ak }}$, A. Favara ${ }^{\text {p }}$, J. Fay ${ }^{\text {y }}$, O. Fedin ${ }^{\text {al }}$, M. Felcini ${ }^{\text {aw }}$, B. Fenyi ${ }^{\text {ar }}$, T. Ferguson ${ }^{\text {ai }}$,

\footnotetext{
' Also supported by CONICET and Universidad Nacional de La Plata, CC 67, 1900 La Plata, Argentina.
} 
F. Ferroni ${ }^{\text {ak }}$, H. Fesefeldt ${ }^{\text {a }}$, E. Fiandrini ${ }^{\text {ah }}$, J.H. Field ${ }^{\text {s }}$, F. Filthaut ${ }^{\text {ai }}$, P.H. Fisher ${ }^{\circ}$, I. Fisk ${ }^{\text {an }}$, G. Forconi ${ }^{\circ}$, L. Fredj ${ }^{\text {s, K. Freudenreich }}{ }^{\text {aw }}$, C. Furetta ${ }^{\text {aa }}$, Yu. Galaktionov ab,o, S.N. Ganguli ', P. Garcia-Abia ${ }^{\text {f }}$, S.S. Gau ${ }^{\text {, }}$ S. Gentile ${ }^{\text {ak }}$, J. Gerald $^{\mathrm{e}}$, N. Gheordanescu ${ }^{\mathrm{m}}, \mathrm{S}$. Giagu ${ }^{\mathrm{ak}}, \mathrm{S}$. Goldfarb ${ }^{\mathrm{v}}$, J. Goldstein k, Z.F. Gong ' , A. Gougas ${ }^{\text {e }}$, G. Gratta ${ }^{\text {ag }}$, M.W. Gruenewald ${ }^{\text {h }}$,

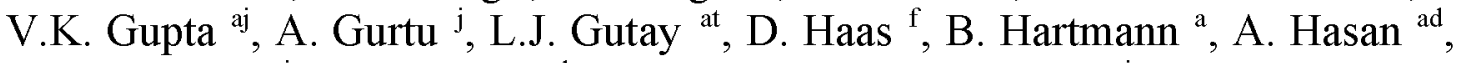
D. Hatzifotiadou ${ }^{i}$, T. Hebbeker ${ }^{\text {h }}$, A. Hervé ${ }^{q}$, J. Hirschfelder ${ }^{\text {ai }}$ W.C. van Hoek ${ }^{\text {ae }}$, H. Hofer ${ }^{\text {aw }}$, H. Hoorani ai, S.R. Hou ${ }^{\text {ay }}$, G. Hu ${ }^{\text {e }}$, V. Innocente ${ }^{q}$, K. Jenkes ${ }^{a}$, B.N. Jin ${ }^{\text {g }}$, L.W. Jones ${ }^{c}$, P. de Jong ${ }^{\mathrm{q}}$, I. Josa-Mutuberria ${ }^{\mathrm{z}}$, A. Kasser ${ }^{\mathrm{v}}$, R.A. Khan ${ }^{\text {r }}$, D. Kamrad ${ }^{\text {av }}$, Yu. Kamyshkov af, J.S. Kapustinsky ${ }^{\mathrm{x}}$, Y. Karyotakis ${ }^{\mathrm{d}}$, M. Kaur ${ }^{\mathrm{r}, 2}$, M.N. Kienzle-Focacci ${ }^{\text {s, D. }}$ Kim ${ }^{\text {ak }}$, D.H. Kim ${ }^{\mathrm{aq}}$, J.K. Kim ${ }^{\mathrm{aq}}$, S.C. Kim ${ }^{\text {aq }}$, W.W. Kinnison ${ }^{\mathrm{x}}$, A. Kirkby ${ }^{\mathrm{ag}}$, D. Kirkby ${ }^{\mathrm{ag}}$, J. Kirkby ${ }^{\mathrm{q}}$, D. Kiss ${ }^{\mathrm{n}}$, W. Kittel ${ }^{\text {ae }}$, A. Klimentov ${ }^{\text {o,ab }}$, A.C. König ${ }^{\text {ae }}$, A. Kopp ${ }^{\text {av }}$, I. Korolko ${ }^{\text {ab }}$, V. Koutsenko ${ }^{\text {o,ab }}$, R.W. Kraemer ${ }^{\text {ai }}$, W. Krenz a , A. Kunin ${ }^{\text {o,ab }}$, P. Lacentre ${ }^{\text {av, },}$, P. Ladron de Guevara ${ }^{\mathrm{z}}$, G. Landi ${ }^{\mathrm{p}}$, C. Lapoint ${ }^{\circ}$, K. Lassila-Perini ${ }^{\mathrm{aw}}$, P. Laurikainen " ${ }^{\text {, }}$ A. Lavorato ${ }^{\mathrm{am}}, \mathrm{M}$. Lebeau ${ }^{\mathrm{q}}$, A. Lebedev ${ }^{\circ}$, P. Lebrun ${ }^{\mathrm{y}}$, P. Lecomte ${ }^{\text {aw }}$, P. Lecoq ${ }^{\text {q }}$, P. Le Coultre ${ }^{\text {aw }}$, H.J. Lee ${ }^{\text {h }}$, C. Leggett ${ }^{c}$, J.M. Le Goff ${ }^{\mathrm{q}}$, R. Leiste ${ }^{\text {av }}$, E. Leonardi ak, P. Levtchenko ${ }^{\text {al }}$, C. Li ${ }^{\mathrm{t}}$, C.H. Lin ${ }^{\text {ay }}$, W.T. Lin ${ }^{\text {ay }}$, F.L. Linde ${ }^{\text {b, }}$, L. Lista ${ }^{\text {ac }}$, Z.A. Liu ${ }^{\mathrm{g}}$, W. Lohmann ${ }^{\text {av }}$, E. Longo ${ }^{\text {ak }}$, W. Lu ${ }^{\text {ag }}$, Y.S. Lu ${ }^{\mathrm{g}}, \mathrm{K}$. Lübelsmeyer ${ }^{\mathrm{a}}$, C. Luci ${ }^{\text {ak }}$, D. Luckey ${ }^{\circ}$, L. Luminari ${ }^{\text {ak }}$, W. Lustermann au, W.G. Ma ${ }^{\text {t }}$, M. Maity ${ }^{\mathrm{j}}$, G. Majumder ${ }^{\mathrm{j}}$, L. Malgeri ${ }^{\text {ak }}$, A. Malinin ${ }^{a b}$, C. Maña ${ }^{z}$, D. Mangeol ${ }^{\text {ae }}$, S. Mangla ${ }^{j}$, P. Marchesini ${ }^{\text {aw }}$, A. Marin ${ }^{\text {k, }}$, J.P. Martin ${ }^{\mathrm{y}}$, F. Marzano ${ }^{\mathrm{ak}}$, G.G.G. Massaro ${ }^{\mathrm{b}}$, D. McNally ${ }^{\mathrm{q}}$, S. Mele ${ }^{\mathrm{q}}$,

L. Merola $^{\text {ac }}$, M. Meschini ${ }^{\text {p }}$,W.J. Metzger ae , M. von der Mey a, Y. Mi v, D. Migani ${ }^{\mathrm{i}}$, A. Mihul ${ }^{\mathrm{m}}$, A.J.W. van Mil ${ }^{\text {ae }}$, H. Milcent ${ }^{\mathrm{q}}$, G. Mirabelli ${ }^{\text {ak }}$, J. Mnich ${ }^{\mathrm{q}}$, P. Molnar ${ }^{\text {h }}$, B. Monteleoni ${ }^{\text {p }}$, R. Moore ${ }^{\text {c }}$, T. Moulik ${ }^{\mathrm{j}}$, R. Mount ${ }^{\mathrm{ag}}$, F. Muheim ${ }^{\mathrm{s}}$, A.J.M. Muijs ${ }^{b}$, S. Nahn ${ }^{\circ}$, M. Napolitano ${ }^{a c}$, F. Nessi-Tedaldi ${ }^{\text {aw }}$, H. Newman ${ }^{\text {ag }}$, T. Niessen ${ }^{\text {a }}$, A. Nippe ${ }^{\text {v }}$, A. Nisati ${ }^{\text {ak }}$, H. Nowak ${ }^{\text {av }}$, Y.D. Oh ${ }^{\text {aq }}$, H. Opitz ${ }^{\text {a }}$, G. Organtini ${ }^{\text {ak }}$, R. Ostonen ", S. Palit ${ }^{1}$, C. Palomares ${ }^{\text {z }}$, D. Pandoulas ${ }^{\text {a }}$, S. Paoletti ak ${ }^{\mathrm{a}}$, P. Paolucci ${ }^{\text {ac }}$, H.K. Park ${ }^{\text {ai }}$, I.H. Park ${ }^{\text {aq }}$, G. Pascale ${ }^{\text {ak }}$, G. Passaleva ${ }^{\text {q }}$, S. Patricelli ${ }^{\text {ac }}$, T. Paul ${ }^{1}$, M. Pauluzzi ah ${ }^{\text {a }}$ C. Paus ${ }^{\text {q }}$, F. Pauss ${ }^{\text {aw }}$, D. Peach ${ }^{\mathrm{q}}$, Y.J. Pei a, S. Pensotti aa, D. Perret-Gallix ${ }^{\text {d }}$, B. Petersen ${ }^{\text {ae }}$, S. Petrak ${ }^{\text {h }}$, A. Pevsner e, D. Piccolo ${ }^{a c}$, M. Pieri ${ }^{p}$, P.A. Piroué aj, E. Pistolesi ${ }^{\text {aa }}$, V. Plyaskin ${ }^{\text {ab }}$, M. Pohl ${ }^{\text {aw }}$, V. Pojidaev ab, p, H. Postema ${ }^{\circ}$, N. Produit ${ }^{\text {s }}$, D. Prokofiev ${ }^{\text {al }}$, J. Quartieri am, G. Rahal-Callot ${ }^{\text {aw }}$, N. Raja ${ }^{\text {j, P.G. Rancoita }}{ }^{\text {aa }}$, M. Rattaggi ${ }^{\text {aa }}$, G. Raven ${ }^{\text {an }}$, P. Razis ${ }^{\text {ad }}$, K. Read ${ }^{\text {af }}$, D. Ren ${ }^{\text {aw }}$, M. Rescigno ${ }^{\text {ak }}$, S. Reucroft ${ }^{1}$, T. van Rhee ${ }^{\text {as }}$,

\footnotetext{
${ }^{2}$ Also supported by Panjab University, Chandigarh-160014, India.
} 
S. Riemann ${ }^{\text {av }}$, K. Riles ${ }^{c}$, O. Rind ${ }^{c}$, A. Robohm ${ }^{\text {aw }}$, J. Rodin ${ }^{\circ}$, B.P. Roe ${ }^{c}$, L. Romero ${ }^{\mathrm{z}}$, S. Rosier-Lees ${ }^{\mathrm{d}}$, Ph. Rosselet ${ }^{\mathrm{v}}$, W. van Rossum ${ }^{\text {as }}, \mathrm{S}$. Roth ${ }^{\text {a }}$, J.A. Rubio ${ }^{\text {q, D. Ruschmeier }}{ }^{\text {h }}$, H. Rykaczewski ${ }^{\text {aw }}$, J. Salicio ${ }^{\text {q }}$, E. Sanchez ${ }^{\text {z }}$,

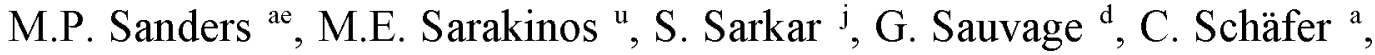
V. Schegelsky ${ }^{\text {al }}$, S. Schmidt-Kaerst ${ }^{\text {a }}$, D. Schmitz ${ }^{\text {a }}$, M. Schneegans ${ }^{\text {d }}$, N. Scholz ${ }^{\text {aw }}$, H. Schopper ${ }^{\text {ax }}$, D.J. Schotanus ${ }^{\text {ae }}$, J. Schwenke a , G. Schwering ${ }^{\text {a }}$, C. Sciacca ${ }^{\text {ac }}$, D. Sciarrino ${ }^{\text {s }}$, L. Servoli ah, S. Shevchenko ${ }^{\text {ag }}$, N. Shivarov ap , V. Shoutko ${ }^{\text {ab }}$, J. Shukla ${ }^{\mathrm{x}}$, E. Shumilov ${ }^{\text {ab }}$, A. Shvorob ${ }^{\text {ag }}$, T. Siedenburg a, D. Son ${ }^{\text {aq }}$, V. Soulimov ${ }^{\text {ac }}$, B. Smith ${ }^{\circ}$, P. Spillantini ${ }^{\text {p }}$, M. Steuer ${ }^{\circ}$, D.P. Stickland ${ }^{\text {aj, }}$, H. Stone aj, B. Stoyanov ap, A. Straessner ${ }^{a}$, K. Sudhakar ${ }^{j}$, G. Sultanov ${ }^{\text {r }}$, L.Z. Sun ${ }^{\mathrm{t}}$, G.F. Susinno ${ }^{\text {s, H. Suter }}{ }^{\text {aw }}$, J.D. Swain ${ }^{r}$, X.W. Tang ${ }^{g}$, L. Tauscher ${ }^{\text {f }}$, L. Taylor ${ }^{1}$, Samuel C.C. Ting ${ }^{\circ}$, S.M. Ting ${ }^{\circ}$, S.C. Tonwar ${ }^{j}$, J. Tóth ${ }^{\mathrm{n}}$, C. Tully aj, H. Tuchscherer ${ }^{\text {ar }}$, K.L. Tung ${ }^{\mathrm{g}}$, Y. Uchida ${ }^{\mathrm{o}}$, J. Ulbricht ${ }^{\mathrm{aw}}$, U. Uwer ${ }^{\mathrm{q}}$, E. Valente ${ }^{\text {ak }}$, G. Vesztergombi ${ }^{n}$, I. Vetlitsky ${ }^{\text {ab }}$, G. Viertel ${ }^{\text {aw }}$, M. Vivargent ${ }^{d}$, S. Vlachos ${ }^{f}$,

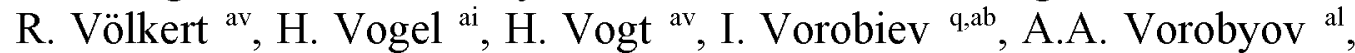
A. Vorvolakos ${ }^{\text {ad }}$, M. Wadhwa ${ }^{\mathrm{f}}$, W. Wallraff ${ }^{\mathrm{a}}$, J.C. Wang ${ }^{\circ}$, X.L. Wang ${ }^{\mathrm{t}}$, Z.M. Wang ${ }^{\mathrm{t}}$, A. Weber ${ }^{\mathrm{a}}$, S.X. Wu ${ }^{\circ}, \mathrm{S}$. Wynhoff ${ }^{\mathrm{a}}$, J. Xu ${ }^{\mathrm{k}}$, Z.Z. Xu ${ }^{\mathrm{t}}$, B.Z. Yang ${ }^{\mathrm{t}}$, C.G. Yang ${ }^{\mathrm{g}}$, X.Y. Yao ${ }^{\mathrm{g}}$, J.B. Ye ${ }^{\mathrm{t}}$, S.C. Yeh ${ }^{\mathrm{az}}$, J.M. You ${ }^{\text {ai }}$, An. Zalite ${ }^{\text {al }}$, Yu. Zalite ${ }^{\text {al }}$, P. Zemp ${ }^{\text {aw }}$, Y. Zeng ${ }^{\text {a }}$, Z. Zhang ${ }^{\text {g }}$, Z.P. Zhang ${ }^{\mathrm{t}}$, B. Zhou ${ }^{\mathrm{k}}$, Y. Zhou ${ }^{c}$, G.Y. Zhu ${ }^{\mathrm{g}}$, R.Y. Zhu ${ }^{\mathrm{ag}}$, A. Zichichi ${ }^{\mathrm{i}, \mathrm{q}, \mathrm{r}}$, F. Ziegler ${ }^{\text {av }}$

${ }^{a}$ I. Physikalisches Institut, RWTH, D-52056 Aachen, FRG ${ }^{4}$

III. Physikalisches Institut, RWTH, D-52056 Aachen, FRG ${ }^{4}$

${ }^{\mathrm{b}}$ National Institute for High Energy Physics, NIKHEF, and University of Amsterdam, NL-1009 DB Amsterdam, The Netherlands

' University of Michigan, Ann Arbor, MI 48109, USA

${ }^{d}$ Laboratoire d'Annecy-le-Vieux de Physique des Particules, LAPP, IN2P3-CNRS, BP 110, F-74941 Annecy-le-Iieux CEDEX, France

e Johns Hopkins University, Baltimore, MD 21218, USA

$\mathrm{f}$ Institute of Physics, University of Basel, $\mathrm{CH}-4056$ Basel, Switzerland

${ }^{\mathrm{g}}$ Institute of High Energy Physics, IHEP, 100039 Beijing, China ${ }^{5}$

${ }^{\mathrm{h}}$ Humboldt University, D-10099 Berlin, FRG ${ }^{4}$

${ }^{\mathrm{i}}$ University of Bologna and INFN-Sezione di Bologna, I-40126 Bologna, Italy

${ }^{\mathrm{j}}$ Tata Institute of Fundamental Research, Bombay 400005 , India

${ }^{\mathrm{k}}$ Boston University, Boston, MA 02215, USA

${ }^{1}$ Northeastern University, Boston, MA 02115, USA

${ }^{\mathrm{m}}$ Institute of Atomic Physics and University of Bucharest, R-76900 Bucharest, Romania

${ }^{n}$ Central Research Institute for Physics of the Hungarian Academy of Sciences, H-1525 Budapest 114, Hungary ${ }^{6}$

- Massachusetts Institute of Technology, Cambridge, MA 02139, USA

${ }^{\mathrm{p}}$ INFN Sezione di Firenze and University of Florence, I-50125 Florence, Italy

${ }^{\mathrm{q}}$ European Laboratory for Particle Physics, CERN, CH-1211 Geneva 23, Switzerland

${ }^{\mathrm{r}}$ World Laboratory, FBLJA Project, CH-1211 Geneva 23, Switzerland

${ }^{\mathrm{s}}$ University of Geneva, $\mathrm{CH}-1211$ Geneva 4, Switzerland

\footnotetext{
${ }^{3}$ Supported by Deutscher Akademischer Austauschdienst.

${ }^{4}$ Supported by the German Bundesministerium für Bildung, Wissenschaft, Forschung und Technologie.

${ }^{5}$ Supported by the National Natural Science Foundation of China.

${ }^{6}$ Supported by the Hungarian OTKA fund under contract numbers T14459, T19181 and T24011.
} 


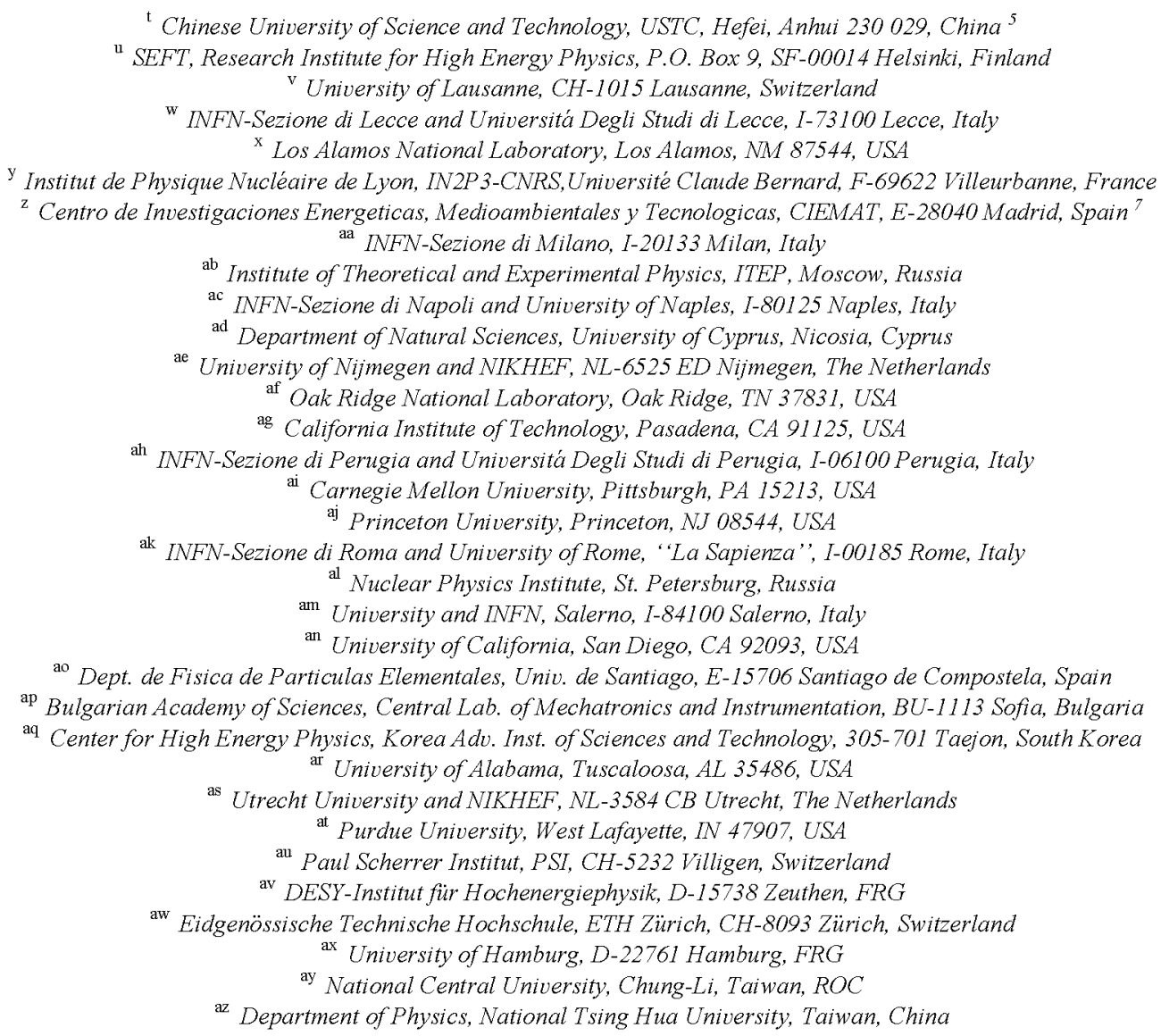

Received 2 February 1998

Editor: K. Winter

\begin{abstract}
Using the data collected by the L3 experiment at LEP from 1991 to 1995 at energies around the Z mass, a measurement of the weak anomalous magnetic dipole moment, $a_{\tau}^{w}$, and of the weak electric dipole moment, $d_{\tau}^{w}$, of the $\tau$ lepton is performed. These quantities are obtained from angular distributions in $\mathrm{e}^{+} \mathrm{e}^{-} \rightarrow \tau^{+} \tau^{-} \rightarrow h^{+} \bar{\nu}_{\tau} h^{-} \nu_{\tau}$, where $h$ is a $\pi$ or a $\rho$. The results are: $\operatorname{Re}\left(a_{\tau}^{w}\right)=(0.0 \pm 1.6 \pm 2.3) \times 10^{-3}, \operatorname{Im}\left(a_{\tau}^{w}\right)=(-1.0 \pm 3.6 \pm 4.3) \times 10^{-3}$ and $\operatorname{Re}\left(d_{\tau}^{w}\right)=(-0.44 \pm 0.88 \pm$ $1.33) \times 10^{-17} e \cdot \mathrm{cm}$. This is the first direct measurement of $a_{\tau}^{w}$. (c) 1998 Elsevier Science B.V. All rights reserved.
\end{abstract}

\section{Introduction}

The weak anomalous magnetic and weak electric dipole moments, $a_{\tau}^{w}$ and $d_{\tau}^{w}$, are intrinsic properties

\footnotetext{
${ }^{7}$ Supported also by the Comisión Interministerial de Ciencia y Technología.
}

of the $\tau$ lepton. In the Standard Model they are zero at Born level but higher order loop corrections lead to $a_{\tau}^{w}=-(2.10+0.61 i) \times 10^{-6}[1]$ and $d_{\tau}^{w} \sim 3 \times$ $10^{-37} e \mathrm{~cm}$ [2]. A measurement of $a_{\tau}^{w}$ or $d_{\tau}^{w}$ significantly different from these predictions would point unambiguously to new physics such as substructure of the $\tau$ [3]. Moreover, CP violation in the $Z \rightarrow \tau^{+} \tau^{-}$ vertex could be manifested in the value of $d_{\tau}^{w}$ [4]. 
Weak dipole moments produce asymmetries in the azimuthal angular distributions of the $\tau$ charged decay products in a coordinate system defined by the $\tau$ direction of flight and the electron beam. We can measure these asymmetries in the channels $\mathrm{e}^{+} \mathrm{e}^{-} \rightarrow$ $\mathrm{Z} \rightarrow \tau^{+} \tau^{-} \rightarrow h^{+} \bar{\nu}_{\tau} h^{-} \nu_{\tau}$, where $h$ is a $\pi$ or a $\rho$, since it is possible to reconstruct the $\tau$ flight direction, up to a twofold ambiguity, for these final states [5].

In this paper we present measurements of the weak anomalous magnetic and weak electric dipole moments of the $\tau$ lepton. The weak electric dipole moment, $d_{\tau}^{w}$, has been measured previously in other experiments [6,7]. This is the first direct measurement of the weak anomalous magnetic moment, $a_{\tau}^{w}$.

\section{Method of the measurement}

In analogy with the electromagnetic dipole moments, the weak dipole moments $a_{\tau}^{w}$ and $d_{\tau}^{w}$ are introduced using the following effective Lagrangian [8]:

$\mathscr{L}_{\mathrm{int}}^{\mathrm{eff}}=-\frac{i}{2} d_{\tau}^{w} \bar{\psi} \omega \sigma^{\mu \nu} \gamma_{5} \psi \mathrm{Z}_{\mu \nu}+\frac{1}{2} \frac{e a_{\tau}^{w}}{2 m_{\tau}} \bar{d} \omega \sigma^{\mu \nu} \psi \mathrm{Z}_{\mu \nu}$

with $Z_{\mu \nu}=\partial_{\mu} Z_{\nu}-\partial_{\nu} Z_{\mu}$.

The cross section for $\mathrm{e}^{+} \mathrm{e}^{-} \rightarrow Z \rightarrow \tau^{+} \tau^{-}$, divided in a spin-independent $\left(\sigma^{0}\right)$ and a spin-dependent part $\left(\sigma^{S}\right)$, can be written $[1,8]$ :

$$
\frac{d \sigma}{d \Omega_{\tau^{-}}}=\frac{d \sigma^{0}}{d \Omega_{\tau^{-}}}+\frac{d \sigma^{S}}{d \Omega_{\tau^{-}}} .
$$

The spin-dependent part reads:

$$
\begin{aligned}
\frac{d \sigma^{s}}{d \Omega_{\tau^{-}}}= & \frac{\alpha^{2} \beta}{128 \sin ^{3} \theta_{\mathrm{W}} \cos ^{3} \theta_{\mathrm{W}} \Gamma_{\mathrm{Z}}^{2}} \\
& \times\left\{\left(s_{-}+s_{+}\right)_{x} X_{+}+\left(s_{-}-s_{+}\right)_{y} Y_{-}\right. \\
& \left.+\left(s_{-}+s_{+}\right)_{y} Y_{+}+\left(s_{-}+s_{+}\right)_{z} Z_{+}\right\} .
\end{aligned}
$$

Here $s_{ \pm}$is the spin vector of the $\tau^{ \pm}$in its rest frame, $\alpha$ is the fine structure constant, $\Gamma_{Z}$ is the $Z$ width, $\gamma=m_{\mathrm{Z}} / 2 m_{\tau}$ where $m_{\mathrm{Z}}$ is the mass of the $Z$ and $m_{\tau}$ is the mass of the $\tau, \beta=\sqrt{1-\left(1 / \gamma^{2}\right)}$ and $\theta_{w}$ is the weak mixing angle. The coefficients $X_{+}$, $Y_{-}, Y_{+}$and $Z_{+}$are given by:

$$
\begin{aligned}
X_{+}= & g_{\mathrm{A}} \sin \theta_{\tau^{-}}\left\{-\left[2 g_{\mathrm{V}}^{2}+\left(g_{\mathrm{V}}^{2}+g_{\mathrm{A}}^{2}\right) \beta \cos \theta_{\tau^{-}}\right]\right. \\
& \times \frac{g_{\mathrm{V}}}{\gamma \sin \theta_{\mathrm{W}} \cos \theta_{\mathrm{W}}} \\
& +2 \gamma\left[2 g_{\mathrm{V}}^{2}\left(2-\beta^{2}\right)+\left(g_{\mathrm{V}}^{2}+g_{\mathrm{A}}^{2}\right) \beta \cos \theta_{\tau^{-}}\right] \\
& \left.\times \operatorname{Re}\left(a_{\tau}^{w \prime}\right)\right\} \\
Y_{-}= & 2 g_{\mathrm{A}} \gamma \beta \sin \theta_{\tau^{-}}\left[2 g_{\mathrm{V}}^{2}+\left(g_{\mathrm{V}}^{2}+g_{\mathrm{A}}^{2}\right) \beta \cos \theta_{\tau^{-}}\right] \\
& \times \frac{2 m_{\tau} \operatorname{Re}\left(d_{\tau}^{w}\right)}{e} ; \\
Y_{+}= & -2 g_{\mathrm{V}} \gamma \beta \sin \theta_{\tau^{-}}\left[2 g_{\mathrm{A}}^{2}+\left(g_{\mathrm{V}}^{2}+g_{\mathrm{A}}^{2}\right) \beta \cos \theta_{\tau^{-}}\right] \\
& \times \operatorname{Im}\left(a_{\tau}^{w}\right) ; \\
Z_{+}= & -\frac{g_{\mathrm{V}} g_{\mathrm{A}}}{\sin \theta_{\mathrm{W}} \cos \theta_{\mathrm{W}}}\left[\left(g_{\mathrm{V}}^{2}+g_{\mathrm{A}}^{2}\right) \beta\left(1+\cos { }^{2} \theta_{\tau^{-}}\right)\right. \\
& \left.+2\left(g_{\mathrm{V}}^{2}+\beta^{2} g_{\mathrm{A}}^{2}\right) \cos \theta_{\tau^{-}}\right]+2 g_{\mathrm{A}}\left[4 g_{\mathrm{V}}^{2} \cos \theta_{\tau^{-}}\right. \\
& \left.+\left(g_{\mathrm{V}}^{2}+g_{\mathrm{A}}^{2}\right) \beta\left(1+\cos ^{2} \theta_{\tau^{-}}\right)\right] \operatorname{Re}\left(a_{\tau}^{w}\right),
\end{aligned}
$$

where $g_{\mathrm{V}}$ and $g_{\mathrm{A}}$ are the neutral current vector and axial-vector coupling constants, respectively and $e$ is the positron charge. The imaginary part of $d_{\tau}^{w}$ is not considered [9].

In the coordinate system of Fig. 1 Eq. (3) can be rewritten $[1,8]$ :

$$
\begin{aligned}
& \frac{d \sigma^{S}\left(\tau^{+} \tau^{-} \rightarrow h^{+} \bar{\nu}_{\tau} h^{-} \nu_{\tau}\right)}{d\left(\cos \theta_{\tau^{-}}\right) d \phi_{h^{ \pm}}} \\
& =\frac{\alpha^{2} \beta \pi}{128 \sin ^{3} \theta_{\mathrm{W}} \cos ^{3} \theta_{\mathrm{W}} \Gamma_{\mathrm{Z}}^{2}} \\
& \quad \times \alpha_{h^{ \pm}}\left(\mp X_{+} \cos \phi_{h^{ \pm}}+\left(Y_{-} \mp Y_{+}\right) \sin \phi_{h^{ \pm}}\right),
\end{aligned}
$$

where $\phi_{h}$ is the azimuthal angle of the hadron and

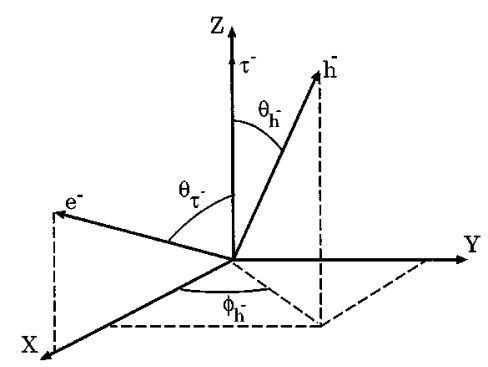

Fig. 1. Reference system used in this analysis. The $z$ axis points in the $\tau$ flight direction and the $x$ axis is fixed by the plane containing the $\tau$ and the electron flight directions. 
$\alpha_{h}$ is the polarisation analysing power [10], which depends on the $\tau$ decay mode.

In order to measure $a_{\tau}^{w}$ and $\operatorname{Re}\left(d_{\tau}^{w}\right)$, the following asymmetries are defined using the angular dependences in Eq. (8):

$$
\begin{aligned}
& A_{c c}^{ \pm}=\frac{\sigma_{c c}^{ \pm}(+)-\sigma_{c c}^{ \pm}(-)}{\sigma_{c c}^{ \pm}(+)+\sigma_{c c}^{ \pm}(-)} ; \\
& A_{s}^{ \pm}=\frac{\sigma_{s}^{ \pm}(+)-\sigma_{s}^{ \pm}(-)}{\sigma_{s}^{ \pm}(+)+\sigma_{s}^{ \pm}(-)} ; \\
& A_{s c}^{ \pm}=\frac{\sigma_{s c}^{ \pm}(+)-\sigma_{s c}^{ \pm}(-)}{\sigma_{s c}^{ \pm}(+)+\sigma_{s c}^{ \pm}(-)},
\end{aligned}
$$

where

$$
\begin{aligned}
& \sigma_{c c}^{ \pm}(+)= \sigma\left(\cos \theta_{\tau^{-}}>0, \cos \phi_{h^{ \pm}}>0\right) \\
&+\sigma\left(\cos \theta_{\tau^{-}}<0, \cos \phi_{h^{ \pm}}<0\right) ; \\
& \sigma_{c c}^{ \pm}(-)= \sigma\left(\cos \theta_{\tau^{-}}>0, \cos \phi_{h^{ \pm}}<0\right) \\
&+\sigma\left(\cos \theta_{\tau^{-}}<0, \cos \phi_{h^{ \pm}}>0\right) ; \\
& \sigma_{s}^{ \pm}(+)= \sigma\left(\sin \phi_{h^{ \pm}}>0\right) ; \\
& \sigma_{s}^{ \pm}(-)=\sigma\left(\sin \phi_{h^{ \pm}}<0\right) ; \\
& \sigma_{s c}^{ \pm}(+)=\sigma\left(\cos \theta_{\tau^{-}}>0, \sin \phi_{h^{ \pm}}>0\right) \\
&+\sigma\left(\cos \theta_{\tau^{-}}<0, \sin \phi_{h^{ \pm}}<0\right) ; \\
& \sigma_{s c}^{ \pm}(-)=\sigma\left(\cos \theta_{\tau^{-}}>0, \sin \phi_{h^{ \pm}}<0\right) \\
&+ \sigma\left(\cos \theta_{\tau^{-}}<0, \sin \phi_{h^{ \pm}}>0\right) .
\end{aligned}
$$

Superscripts \pm indicate the charge of the $\tau$, while + or - signs in parenthesis indicate angular regions. Subscripts indicate combinations of sines and cosines [8]. These asymmetries are directly related to the weak dipole moments as given below:

$$
\begin{aligned}
& A_{c c}^{\pi^{\mp}}= \pm \delta_{c c}^{\pi} \pm \kappa_{c c}^{\pi} \operatorname{Re}\left(a_{\tau}^{w}\right), \\
& A_{s}^{\pi^{\mp}}= \pm \kappa_{s}^{\pi} \operatorname{Im}\left(a_{\tau}^{w}\right), \\
& A_{s c}^{\pi^{\mp}}=-\kappa_{s c}^{\pi} \frac{2 m_{\tau}}{e} \operatorname{Re}\left(d_{\tau}^{w}\right) ; \\
& A_{c c}^{\rho^{\mp}}= \pm \delta_{c c}^{\rho} \pm \kappa_{c c}^{\rho} \operatorname{Re}\left(a_{\tau}^{w}\right), \\
& A_{s}^{\rho^{\mp}}= \pm \kappa_{s}^{\rho} \operatorname{Im}\left(a_{\tau}^{w}\right), \\
& A_{s c}^{\rho^{\mp}}=-\kappa_{s c}^{\rho} \frac{2 m_{\tau}}{e} \operatorname{Re}\left(d_{\tau}^{w}\right) .
\end{aligned}
$$

The coefficients $\delta_{i}^{h}$ and $\kappa_{i}^{h}$ with $i=c c, s, s c$ are obtained by integration of Eq. (2) over the angular regions given in Eq. (10). The quantities $\kappa_{i}^{h}$ are typically of the order of 10 , while $\delta_{i}^{h}$ are $10^{-5} \kappa_{i}^{h}$. The latter are therefore neglected in this measurement. The values of $\Gamma_{\mathrm{Z}}, m_{\mathrm{Z}}, m_{\tau}, g_{\mathrm{V}}, g_{\mathrm{A}}$ and $\sin ^{2} \theta_{W}$ are taken from Ref. [11].

\subsection{Flight direction reconstruction of the $\tau$}

The angle $\phi_{h}$ is obtained by reconstructing the $\tau$ direction of flight. An illustration of the event kinematics is shown in Fig. 2. Assuming that the $\tau$ leptons are produced back-to-back and their energies are $E_{\tau}=m_{\mathrm{Z}} / 2$, the angle between the hadron $h^{ \pm}$ and the $\tau^{ \pm}$is:

$$
\cos \theta_{h^{ \pm} \tau^{ \pm}}=\frac{m_{\mathrm{Z}} E_{h^{ \pm}}-m_{\tau}^{2}-m_{h^{ \pm}}^{2}}{\sqrt{\left(m_{Z}^{2}-4 m_{\tau}^{2}\right)\left(E_{h^{ \pm}}^{2}-m_{h^{ \pm}}^{2}\right)}} .
$$

If we define the unit vectors:

$$
\begin{aligned}
& \hat{n}_{1}=\hat{n}_{2} \times \hat{n}_{3}=\frac{1}{\sin \psi}\left(\hat{p}_{h^{-}}-\hat{p}_{h^{+}} \cos \psi\right) \\
& \hat{n}_{2}=\frac{1}{\sin \psi}\left(\hat{p}_{h^{-}} \times \hat{p}_{h^{+}}\right) \\
& \hat{n}_{3}=-\hat{p}_{h^{+}},
\end{aligned}
$$

where $\cos \psi=\hat{p}_{h^{-}} \cdot \hat{p}_{h^{+}}$, and $\hat{p}_{h^{ \pm}}$are unit vectors in

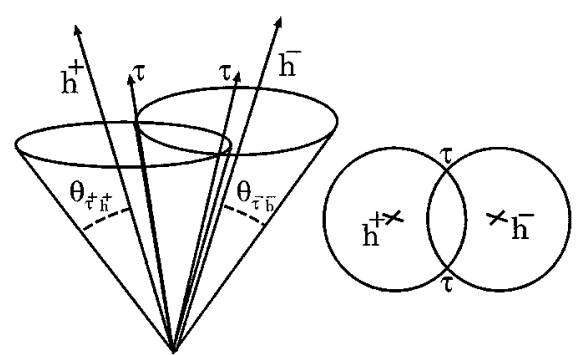

Fig. 2. Geometric view of the $\tau$ flight direction reconstruction. Each of the hadron directions defines a cone of possible $\tau$ directions. The construction involves reflecting one cone in the plane normal to the $\tau$ direction. Requiring that the $\tau$ 's be produced back-to-back allows one to solve for the $\tau$ direction up to a twofold ambiguity. 
the direction of the hadron $h^{ \pm}$momenta, the $\tau$ direction can be written $\hat{e}_{\tau}=a \hat{n}_{1}+b \hat{n}_{2}+c \hat{n}_{3}$, where $a=\frac{1}{\sin \psi}\left(\cos \theta_{h^{-} \tau^{-}}+\cos \theta_{h^{+} \tau^{+}} \cos \psi\right) ;$

$c=\cos \theta_{h^{+} \tau^{+}}$

$b= \pm \sqrt{1-a^{2}-c^{2}}$.

The ambiguity in the $\tau$ direction reconstruction is reflected in the sign ambiguity of the $b$ parameter.

\section{Selection of the data}

This analysis uses the complete data sample collected by L3 from 1991 to 1995 at energies around the $\mathrm{Z}$ mass, corresponding to an integrated luminosity of $150 \mathrm{pb}^{-1}$. The $\mathrm{L} 3$ detector is described elsewhere [12]. The analysis is restricted to events with $\left|\cos \theta_{\text {thrust }}\right|<0.7$. A preselection of leptonic $Z$ decays is done, requiring low multiplicity events with backto-back topology. This preselection rejects backgrounds such as hadronic $Z$ decays, two-photon events and beam-gas interactions. Each event is divided in two hemispheres by a plane perpendicular to the thrust axis. Hadronic $\tau$ decays are identified by requiring, in each hemisphere, a track in the central tracking detector pointing to an energy deposition in the calorimeters which is not consistent with an electromagnetic shower or a minimum ionizing particle in the hadron calorimeter. Then an algorithm [13] is applied to determine the number of neutral electromagnetic showers and their energies. Two distinct neutral electromagnetic showers form a $\pi^{0}$ candidate if their invariant mass is within $40 \mathrm{MeV}$ of the $\pi^{0}$ mass. A single neutral electromagnetic shower forms a $\pi^{0}$ candidate if its energy exceeds 1 $\mathrm{GeV}$. Its transverse energy profile must be consistent with a single electromagnetic shower or a twophoton hypothesis for which the invariant mass is within $50 \mathrm{MeV}$ of the $\pi^{0}$ mass.

The $\tau^{-} \rightarrow \pi^{-} \nu_{\tau}{ }^{8}$ selection admits no $\pi^{0}$ candidates and no neutral showers with energy greater than $0.5 \mathrm{GeV}$. The calorimetric energy deposition must be consistent with the measured track momentum.

\footnotetext{
${ }^{8}$ No distinction between charged pions and kaons is made. Charge conjugate decays are considered by implication.
}

Table 1

Efficiencies and backgrounds for the selected sample inside the fiducial volume

\begin{tabular}{llll}
\hline Final State & Number of events & Efficiency (\%) & Background (\%) \\
\hline (i) & 3703 & 43 & 25 \\
(ii) & 3783 & 44 & 24 \\
(iii) & 1152 & 51 & 24 \\
\hline
\end{tabular}

To select $\tau^{-} \rightarrow \rho^{-} \nu_{\tau}$ decays, exactly one $\pi^{0}$ candidate is required in the hemisphere. The invariant mass of the $\pi \pi^{0}$ system must be between $0.45 \mathrm{GeV}$ and $1.2 \mathrm{GeV}$ and its energy must be larger than 5 $\mathrm{GeV}$.

A total of 8638 events is selected and classified as one of the following final states:

(i) $\mathrm{e}^{+} \mathrm{e}^{-} \rightarrow \tau^{+} \tau^{-} \rightarrow \rho^{+} \bar{\nu}_{\tau} \rho^{-} \nu_{\tau}$;

(ii) $\mathrm{e}^{+} \mathrm{e}^{-} \rightarrow \tau^{+} \tau^{-} \rightarrow \pi^{+} \bar{\nu}_{\tau} \rho^{-} \nu_{\tau}$ or $\rho^{+} \bar{\nu}_{\tau} \pi^{-} \nu_{\tau}$;

(iii) $\mathrm{e}^{+} \mathrm{e}^{-} \rightarrow \tau^{+} \tau^{-} \rightarrow \pi^{+} \bar{\nu}_{\tau} \pi^{-} \boldsymbol{\nu}_{\tau}$.

The number of events, the efficiency and the background fraction for each channel are quoted in Table 1. The efficiency and the background fraction are determined from a Monte Carlo sample [14,15], which is passed through the full detector simulation, reconstruction and selection procedure. The background arises from misidentified $\tau$ decays. The non- $\tau$ background is negligible.

\section{Analysis}

The algorithm for the $\tau$ flight direction reconstruction described previously is applied to the selected data and Monte Carlo samples. The samples are divided into two subsets, the first consisting of events taken between 1991 and 1993 and the second taken in 1994 and 1995. This is done because the
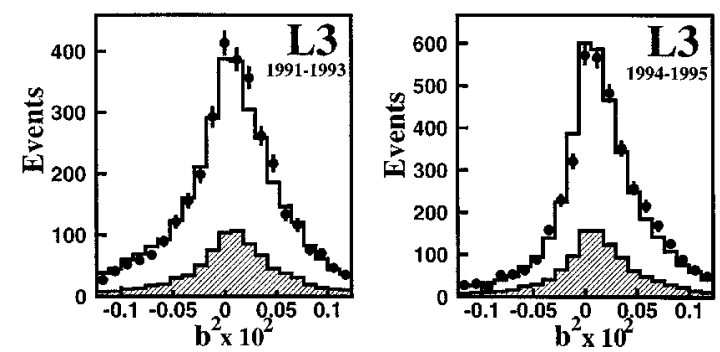

Fig. 3. Distribution of the parameter $b^{2}$ for data (dots) and Monte Carlo (histogram). The background is shown as hatched histograms. 

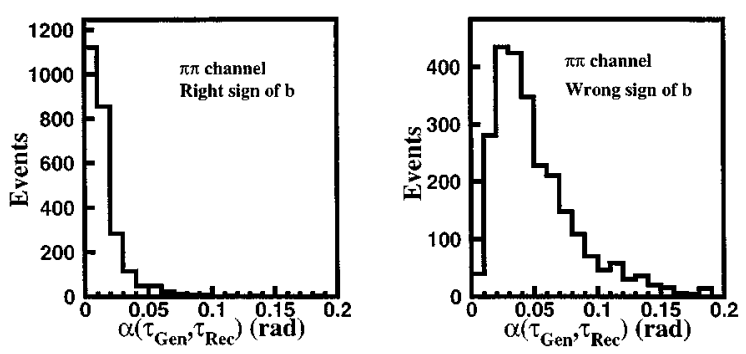

Fig. 4. Reconstruction of the $\tau$ flight direction for $\pi-\pi$ events. The left plot shows the distribution of the angle $\alpha$ between the reconstructed and the generated $\tau$ direction when the ambiguity is solved properly. The right plot shows the same distribution when the wrong solution is taken.

Silicon Microvertex Detector [16] has been available since 1994, allowing for a more precise measurement of tracks.

The reconstruction algorithm can give zero, one or two solutions, which correspond to $b^{2}<0, b^{2}=0$ or $b^{2}>0$, respectively. The case of no solution occurs because of finite detector resolution or because the initial/final state radiation distorts the back-to-back topology. In this case, the event is not used in the analysis. The fraction of reconstructed events is $55 \%$ for the 1991-1993 sample and $64 \%$ for the 1994-1995 sample. Fig. 3 shows the distributions of the $b^{2}$ parameter in both samples for data and Monte Carlo. The good agreement shows that the efficiency and the resolution in the reconstruction is the same for data and Monte Carlo.

The resolution in the reconstruction of the $\tau$ flight direction has been studied using Monte Carlo events and is shown in Fig. 4 for the $\pi-\pi$ channel. In this analysis, for most of the events, the two possible $\tau$ flight directions are used, each contributing with weight $1 / 2$. In the data sample from 94-95, the ambiguity in $b$ is solved [17] for the $\pi-\pi$ subsample with an efficiency varying between $80 \%$ and $60 \%$ for low and high track momentum, respectively. The ambiguity in the $\tau$ flight direction and the influence of the resolution of the detector change the theoretical relations of Eq. (11). This effect is taken into account in the following way. First, a Monte Carlo study is performed, to obtain the resolution function for $\phi_{h}$. Modified expressions are then obtained by convoluting this resolution function with the theoretical cross section of Eq. (8), and taking into account the cross feed between channels (i), (ii), and (iii) and other background:

$$
\left(\begin{array}{c}
A_{c c} \\
A_{s c} \\
A_{s}
\end{array}\right)=\mathscr{M}\left(\begin{array}{c}
\operatorname{Re}\left(a_{\tau}^{w}\right) \\
m_{\tau} d_{\tau}^{w} / e \\
\operatorname{Im}\left(a_{\tau}^{w}\right)
\end{array}\right)
$$

with a different matrix $\mathscr{M}$ for each decay channel [18]. The effects of the detector resolution and of the ambiguity in the $\tau$ flight direction slightly reduce the sensitivity of the measurement of the weak dipole moments. As the off-diagonal terms of $\mathscr{M}$ are not zero, there is a small mixing among the weak dipole moments in the asymmetries.

This analysis has been checked with Monte Carlo samples corresponding to large values of the weak dipole moments. After applying the complete procedure, the input values were recovered.

\section{Systematic errors}

The main sources of systematic errors in the asymmetries are the reconstruction of the $\tau$ flight direction and the selection criteria.

Table 2

Breakdown of the systematic error $\Delta \mathrm{A}$ on the azimuthal asymmetries $\mathrm{A}_{\mathrm{cc}}, \mathrm{A}_{\mathrm{s}}$ and $\mathrm{A}_{\mathrm{sc}}$

\begin{tabular}{llll}
\hline & Source & $\Delta \mathrm{A}$ & \\
\cline { 2 - 4 } & & $1991-1993$ & $1994-1995$ \\
\hline \multirow{2}{*}{ Channel $\tau \rightarrow \rho \nu_{\tau}$} & $\tau$ Direction Reconstruction & 0.013 & 0.010 \\
& Selection cuts & 0.009 & 0.013 \\
& Finite MC Statistics & 0.003 & 0.002 \\
Channel $\tau \rightarrow \pi \nu_{\tau}$ & $\tau$ Direction Reconstruction & 0.026 & 0.024 \\
& Selection cuts & 0.011 & 0.015 \\
& Finite MC Statistics & 0.004 & 0.004 \\
\hline
\end{tabular}




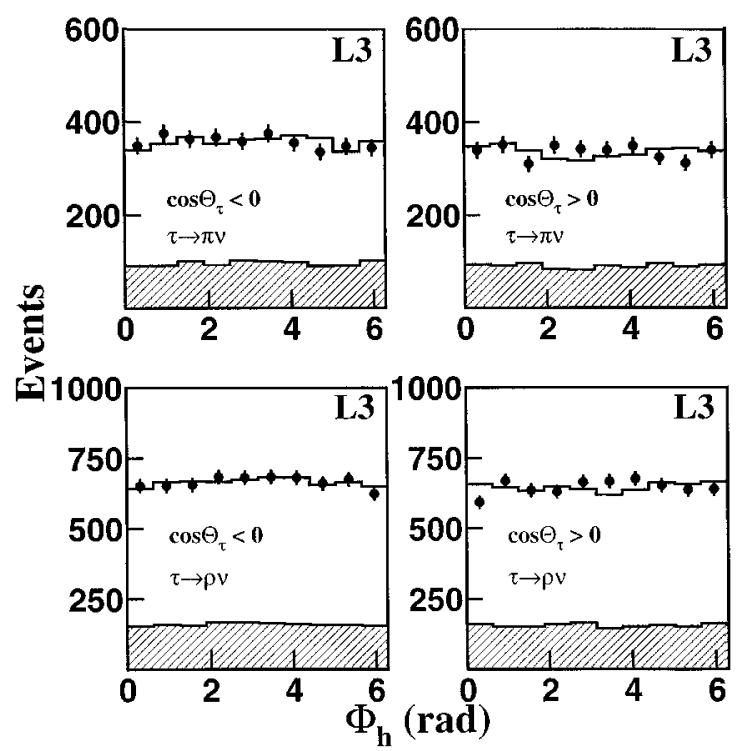

Fig. 5. Comparison between data (dots) and the Standard Model Monte Carlo expectations (histograms) for the $\phi_{h}$ angular distributions in the regions of $\cos \theta_{\tau}$ used to determine the asymmetries. The background is shown as hatched histograms.

The systematic error associated to the reconstruction of the $\tau$ direction arises from four sources: the uncertainties in the resolution functions, the detector homogeneity, the detector alignment with respect to the electron beam and photon radiation. To obtain the contribution due to the resolution, the uncertainty in the width of the resolution functions has been propagated to the asymmetries. This amounts to $0.013(0.026)$ for $1991-1993$ sample and $0.010(0.024)$ for the 1994-1995 sample in the $\rho(\pi)$ channel. The homogeneity of the detector has been studied using a dimuon sample which is known to have a back-to-back topology. This analysis was done in steps of the azimuthal and polar angle and no distortion in the detector was observed. The contribution to the systematic error is determined to be $0.002(0.003)$ in the $\rho(\pi)$ channel. The alignment of the detector with respect to the electron beam was studied using radiative dimuon events and found to be perfect. The effect of photon radiation has been estimated by using the KORALZ generator [14]. Two independent samples have been generated, one of them including photon radiation and the other one not including it. The difference in the asymmetries obtained using these two samples, of $0.001(0.002)$ in the $\rho(\pi)$ channel, is assigned as the systematic error.

To estimate the contribution to the systematic error of the selection, the cuts have been varied within $20 \%$ and the fraction of background has been varied within one standard deviation.

Finally, we took account for the effect of limited Monte Carlo statistics on the efficiencies. No charge dependence of the efficiencies was observed. The estimated systematic errors are given in Table 2 . These values apply to all the asymmetries.

\section{Results and conclusions}

Distributions in the azimuthal angle $\phi_{h}$ for the selected events are shown in Fig. 5 for the complete data sample. The measured values for the asymmetries $A_{c c}, A_{s c}, A_{s}$ are given in Table 3.

Table 3

Measured azimuthal asymmetries for each channel. The first error is statistical and the second is systematic

\begin{tabular}{lrrrr}
\hline & \multicolumn{1}{l}{$\mathrm{A}_{\mathrm{cc}}$} & \multicolumn{1}{c}{$\mathrm{A}_{\mathrm{sc}}$} & \multicolumn{1}{c}{$\mathrm{A}_{s}$} \\
\hline$\tau^{-} \rightarrow \rho^{-} \nu_{\tau}$ & $91-93$ & $0.005 \pm 0.021 \pm 0.016$ & $-0.009 \pm 0.021 \pm 0.016$ & $-0.016 \pm 0.021 \pm 0.016$ \\
& $94-95$ & $0.000 \pm 0.018 \pm 0.016$ & $0.036 \pm 0.018 \pm 0.016$ & $-0.009 \pm 0.018 \pm 0.016$ \\
& & & & $0.004 \pm 0.020 \pm 0.016$ \\
$\tau^{+} \rightarrow \rho^{+} \nu_{\tau}$ & $91-93$ & $0.027 \pm 0.020 \pm 0.016$ & $-0.010 \pm 0.020 \pm 0.016$ & $-0.015 \pm 0.018 \pm 0.016$ \\
& $94-95$ & $0.028 \pm 0.018 \pm 0.016$ & $-0.008 \pm 0.018 \pm 0.016$ & $-0.015 \pm 0.029 \pm 0.028$ \\
$\tau^{-} \rightarrow \pi^{-} \nu_{\tau}$ & $91-93$ & $0.044 \pm 0.029 \pm 0.028$ & $0.032 \pm 0.029 \pm 0.028$ & $0.011 \pm 0.024 \pm 0.028$ \\
& $94-95$ & $-0.019 \pm 0.024 \pm 0.028$ & $-0.010 \pm 0.024 \pm 0.028$ & $0.022 \pm 0.029 \pm 0.028$ \\
$\tau^{+} \rightarrow \pi^{+} \nu_{\tau}$ & $91-93$ & $-0.028 \pm 0.029 \pm 0.028$ & $0.038 \pm 0.029 \pm 0.028$ & $0.031 \pm 0.025 \pm 0.028$ \\
& $94-95$ & $0.029 \pm 0.025 \pm 0.028$ & $-0.024 \pm 0.025 \pm 0.028$ & \\
\hline
\end{tabular}


The values of the weak dipole moments are obtained by solving Eqs. (15). Combining the results for each weak moment taking systematic errors and their correlations into account yields the final results:

$$
\begin{aligned}
& \operatorname{Re}\left(a_{\tau}^{w}\right)=(0.0 \pm 1.6 \pm 2.3) \times 10^{-3} \\
& \operatorname{Im}\left(a_{\tau}^{w}\right)=(-1.0 \pm 3.6 \pm 4.3) \times 10^{-3} \\
& \operatorname{Re}\left(d_{\tau}^{w}\right)=(-0.44 \pm 0.88 \pm 1.33) \times 10^{-17} e \cdot \mathrm{cm},
\end{aligned}
$$

where the first error is statistical and the second is systematic. The values are compatible with the predictions of the Standard Model. The limits at 95\% C.L. are $\left|\operatorname{Re}\left(a_{\tau}^{w}\right)\right|<4.5 \times 10^{-3}, \quad\left|\operatorname{Im}\left(a_{\tau}^{w}\right)\right|<9.9 \times$ $10^{-3},\left|\operatorname{Re}\left(d_{\tau}^{w}\right)\right|<3.0 \times 10^{-17} e \cdot \mathrm{cm}$.

\section{Acknowledgements}

We wish to express our gratitude to the CERN accelerator divisions for the excellent performance of the LEP machine. We acknowledge the effort of all engineers and technicians who have participated in the construction and maintenance of the experiment.

\section{References}

[1] J. Bernabéu, G.A. González-Sprinberg, M. Tung, J. Vidal, Nucl. Phys. B 436 (1995) 474.

[2] M.J. Booth, University of Chicago Report No. EFI-93-02, hep-ph $/ 9301293$.
[3] M.C. González-García, S.F. Novaes, Phys. Lett. B 389 (1996) 707 [CERN-TH /96-185].

[4] W. Bernreuther, U. Löw, J.P. Ma, O. Nachtmann, Z. Phys. C 43 (1989) 117; W. Bernreuther, O. Nachtmann, Phys. Rev. Lett. 63 (1989) 2787

[5] H. Kühn, F. Wagner, Nucl. Phys. B 236 (1984) 16.

[6] ALEPH Collaboration, D. Buskulic et al., Phys. Lett. B 346 (1995) 371

[7] OPAL Collaboration, K. Ackerstaff et al., Z. Phys. C 74 (1997) 403.

[8] J. Bernabéu, G.A. González-Sprinberg, J. Vidal, Phys. Lett. B 326 (1994) 168; U. Stiegler, Z. Phys. C 57 (1993) 511.

[9] J. Bernabéu, private communication.

[10] K. Hagiwara, A.D. Martin, D. Zeppenfeld, Phys. Lett. B 235 (1990) 198

[11] L3 Collaboration, M. Acciarri et al., Z. Phys. C 62 (1994) 551. Particle Data Group, R.M. Barnett et al., Phys. Rev. D 54 (1996) 1 [URL: http://pdg.lbl.gov/].

[12] L3 Collaboration, B. Adeva et al., Nucl. Instr. and Meth. A 289 (1990) 35; O. Adriani et al., Nucl. Instr. and Meth. A 302 (1991) 53; K. Deiters et al., Nucl. Instr. and Meth. A 323 (1992) 162; J.A. Bakken et al., Nucl. Instr. and Meth. A 275 (1989) 81.

[13] L3 Collaboration, O. Adriani et al., Phys. Lett. B 294 (1992) 466.

[14] S. Jadach, B.F.L. Ward, Z. Wąs, Comput. Phys. Commun 66 (1991) 276

[15] The L3 detector simulation is based on GEANT Version 3.15; see R. Brun et al., GEANT 3, CERN DD/EE/84-1 (Revised), September 1987; The GHEISHA program (H. Fesefeldt, RWTH Aachen Report PYTHIA 85/02 (1985)) is used to simulate hadronic interactions.

[16] L3 Collaboration, O. Adriani et al., Nucl. Instr. and Meth. A 351 (1994) 300.

[17] J.H. Kühn, Phys. Lett. B 313 (1993) 458.

[18] E. Sánchez, Ph.D. Thesis, Universidad Complutense de Madrid, 1997. 